\title{
CRISPR-Cas9 in agriculture: Approaches, applications, future perspectives, and associated challenges
}

Prabin Adhikari*, Mousami Poudel

Agriculture and Forestry University

*Corresponding Author Email: adhikariprabin8@gmail.com

\section{Doi: 10.2478/mjhr-2020-0002}

\section{Abstract:}

The discovery of an adaptive immune system especially in archae and bacteria, CRISPR/Cas has revolutionized the field of agriculture and served as a potential gene editing tool, producing great excitement to the molecular scientists for the improved genetic manipulations. CRISPR/Cas9 is a RNA guided endonuclease which is popular among its predecessors ZFN and TALEN's. The utilities of CRISPR from its predecessors is the use of short RNA fragments to locate target and breaking the double strands which avoids the need of protein engineering, thus allowing time efficiency measure for gene editing. It is a simple, flexible and highly efficient programmable DNA cleavage system that can be modified for widespread applications like knocking out the genes, controlling transcription, modifying epigenomes, controlling genome-wide screens, modifying genes for disease and stress tolerance and imaging chromosomes. However, gene cargo delivery system, off target cutting and issues on the safety of living organisms imposes major challenge to this system. Several attempts have been done to rectify these challenges; using sgRNA design software, cas 9 nickases and other mutants. Thus, further addressing these challenges may open the avenue for CRISPR/cas9 for addressing the agriculture related problems.

Keywords: nickases, endonuclease, gene editing, sgRNA binding

\subsection{Introduction:}

In the current scenario, world population is increasing in geometric proportion but the food materials available are increasing in arithmetic scale. By 2050 the world population is expected to reach around ten billions; just 3 decades far away from us [1], [2]. Increasing world population along with the adverse effect of climate change has threatened the world in terms of food security. The situation will be even exacerbated with the decrease in fertile land coupled with reducing yields. By the recent statistics of International Rice Research Institute, it is found that with one hectare of the fertile land per 7.7 seconds is being lost and the effect may even be highly pronounced if the accelerating rate of global temperature prevails [3]. To meet the food security in global scale, the yield potential of the crops grown should nearly be doubled and to obtain this goal, highly adaptable crop varieties to biotic and abiotic stress should be introduced urgently [4], [5].

The development and improvement of crop varieties has been carried out using conventional breeding techniques like hybridization and mutational breeding which has improved crop production to some extent. Genetic manipulation technique has been used for few decades using physical, chemical and biological mutagenesis to study the role of genes and identifying the biological mechanisms for crop improvement. Transgenic techniques have been used further for understanding the plant biology and crop improvement. Despite using these techniques, satisfaction has never been obtained due to the presence of some negative issues[6]. The actual yield seems to be approaching a plateau in next few years and yield stagnation has been currently reported in major cereal crops like rice in East Asia, maize in South Europe and wheat in Northwest Europe[7], [8]. Moreover, the integration of transgene into host genome is non-specific and a matter of public concern when it comes to the edible crop species. So, as to remove these limitations, the use of biotechnology in crop improvement is of utmost importance [8].

In recent years, the use of sequence-specific nucleases (SSNs) has been extensively applied for precise genome editing in crop species [9]. These SSNs create breakage of double strands in the target DNA. The DNA then gets repaired through either non homologous end joining (NHEJ) or homology-directed recombination (HDR) pathways; the former being the more common one; resulting into insertions/deletions and substitution mutations in the target DNAs respectively[10]. The genome editing methods offers a huge advantage in producing defined mutants unlike that of transgenic approaches with random insertions leading to random phenotypes. Genome edited plants also carry their edited DNA for the desired traits, which offers an additional advantage[11]. These improved crops can be used in breeding programs and resulting varieties can be used directly with relatively lesser consumption issues and lesser regulatory procedures as compared to transgenic crops/ conventional genetically modified (GM) crops[12]. This review discusses the applications of recently advanced genome editing tool CRISPR/Cas9 in crop improvement along with the future perspective and associated challenges.

\subsection{Gene editing tools: Engineered nucleases}

When a non-specific nuclease domain is fused with sequence-specific DNA binding domain, then it is said to be engineered. Such fused nuclease has the capacity to cleave the target gene and the breaks are repaired either by NHEJ or HDR, and the whole process is given the term genome editing[13]. Naturally inspired existing technologies for genome engineering include several nucleases like Zinc finger nucleases (ZFNs), Transcription activator-like effector nucleases (TALENs) and CRISPR/Cas9 system. ZFNs and TALENs involve tedious procedure with long turn-over time, costly, and are less reliable as compared to the second generation CRISPR/Cas9 system[14]. More than $\$ 5000$ per pair of commercial ZFNs [15] or TALENs makes it much more expensive. Moreover, the off target activity/toxicity and base skipping activity are higher than CRISPR/cas9. Besides this, long turn-over time has made it difficult for ZFNs and TALENs to scale up. Usually, ZFNs take several weeks to build a few pair for experts and TALENs take about a week to build a few pairs[14] which make it more time consuming than CRISPR/Cas9[16].

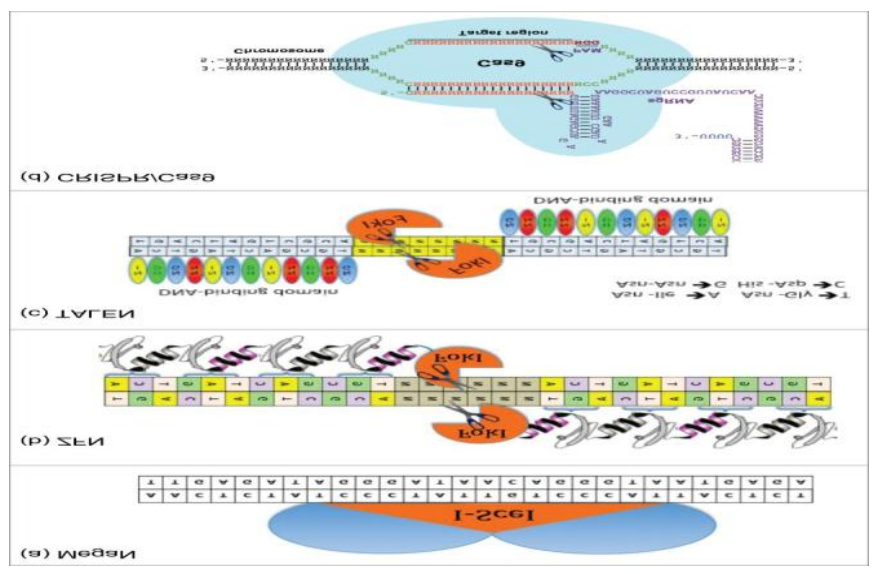

Figure 1: Genome editing tools (SSNs) 


\subsection{CRISPR/Cas9: An advanced genome editing tool}

Clustered Regularly Interspaced Short Palindromic Repeats (CRISPR) is a microbial adaptive immune system which was first identified in E.coli [17]. It consists of two main parts: short identical repeats of 20-40 ladders and a spacer DNA which is not identical. It was known in 80's or 90's that the each set of spacer DNA is unique[18], [19]. In 2000's it was identified that the Spacer DNA matches with the viral bacteriophases[17]. At the same time the number of genes associated with CRISPR was also identified. They are CRISPR associated or Cas genes. Cas genes make Cas proteins. Cas proteins are the helicases that unwind DNA, and nucleases that cut the DNA. These proteins are immune system for bacteria where they could resist bacteriophases[10].

When the bacteriophase injects its DNA what normally would have happen if it does not have immune system is this DNA would hijack the cell and could become invaded in the genome. For more importantly it would make the bunch of these bacteriophases and eventually kill the cell. Since it has a CRISPR system what it is going to do is transcribe and translate protein. So this Cas complex and also transcribe DNA to make crRNA and it will fit right into the protein. It is a way to fight out viral RNA that essentially breaks it apart[20]. In fact, before the infection starts, infection essentially has ended. But what happens when CRISPR do not have spacer that matches? Well, the CRISPR-Cas system works there as well. It's going to create a different class of protein: a class I Cas protein, it takes the DNA in and breaks it apart, and more importantly takes that DNA and copies it into the CRISPR system[21]. CRISPR is in fact a bunch of spacer-repeats but the spacers are essentially history of old infections that won't be infecting again[22].

What scientists thought is if they could hijack this CRISPR system, they could perhaps use it as it is a living cell, so as to either inactivate genes or may be even invade new genes. The research was on and CRISPR/Cas9 was developed. It is identified in labs of Jennifer Doudna and Emmanuelle Charpentier and what she was working on was Streptococcus pyogenes and their CRISPR/Cas system. They only had one Cas protein Cas9. If we look at its major structure, it has nucleases so as to cut the section right there. In S.pyogenes they are also creating two long strips of RNA; CRISPR RNA (crRNA) that gets fit into the Cas and transactivating CRISPR RNA (tracrRNA) that holds the crRNA in place[19]. The whole thing forms the complex where it can break the DNA. It would not be possible to apply if the whole system was not modified. Using one Cas9 protein but putting on the own sequence of DNA (crRNA) and connecting these two together forms a simple system and that was what they actually did. They created a tracrRNA-crRNA chimera. They created a new type of RNA that has a system which is really simple containing two parts; one is the Cas9 protein and the other is the chimera. In simple words, the chimera is also called as guide RNA (gRNA)[23], [24]. CRISPR part and Cas9 part thus together forms a complex and work together. RNA that gets the information at the target site and the protein that actually does the work of cutting.

When the viral DNA or the DNA that we want to cut enters, then it creates a guide RNA that would be complementary to the target DNA and when the DNA starts feeding, the Double strand break (DSB) occurs (Fig2); it gets cut into two pieces, becomes inactive and fixes itself by NHEJ or HDR by the insertion/deletions and substitution respectively. To insert the desired host DNA, host DNA is added and the DNA gets fixed[25].

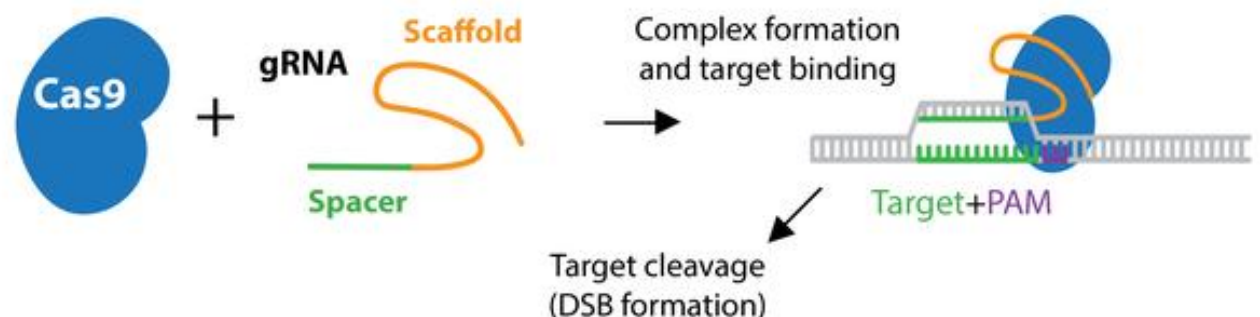

Non-homologous end joining (NHEJ)

\section{WT \\ Insertion

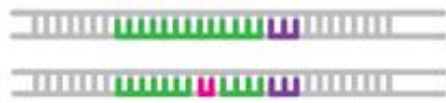 \\ Deletion

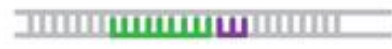 \\ Frameshift}

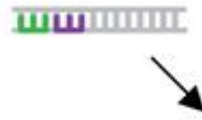

Homology directed repair (HDR)

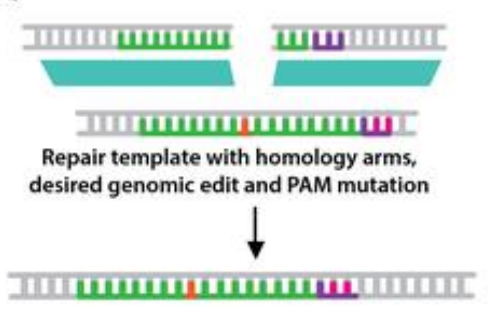

Figure 1: CRISPR/Cas9 for DSBs and repair either by NHEJ or HDR

\subsection{Development and modifications of CRISPR/Cas9}

The most common type of cas9 protein is from the bacteria Streptococcus pyogenes (SpcAs9) which recognizes the NGC-type PAM[26]. This type of PAM sequence is widely distributed throughout plant genomes but it actually does not cover the entire plant genome so this may increase the off target cleavage due to complexing of gRNA with the mismatched complementary target DNA within the genome. Thus several attempts and modifications have been done on Cas9 enzyme for achieving the target specificity and reducing the frequency of off target effects which is shown in Table 1 [8]. An increase in the protospacer adjacent motif length is a strategy that has been used to minimize the off-target cleavage. The CRISPR/Cas system developed from the bacteria other than S.pyogenes like Neisseria meningitidis known as Nmecas9, recognizes an 8-mer PAM sequence (5'-NNNNGATT) that can improve target specificity and reduce potential off-target cleavage [27]while Staphylococcus aureus, Sacas9 recognizes a 6-mer PAM sequence (5'-NNGRRT; [28]. Similarly, Cas9 nuclease activity modifications have also been successful in expanding the applications of CRISPR/Cas9. First, with a modified Cas9 cleavage domain at Cas9-D10A or Cas9-H840A and on combining with paired guide RNAs, Cas9 can cleave a targeted region on the opposite DNA strand, improving its specificity 100-1500-folds. Second, the Cas9 created by point mutation in RuvC and HNH nuclease domains called dead Cas9, or dCas9[29] or CRISPRi become catalytically inactive and thus cannot cleave targeted regions. The co-expression of dCas 9 and a specific sgRNA in the coding region of a gene can prevent the transcription elongation process which may lead to the loss of function of incompletely translated proteins [30]. This approach can be used to block transcription initiation by binding to the operator or the promoter of a gene, such as a transcription factor binding site or RNA polymerase binding site. Such binding can markedly decrease gene expression. The dCas9 can be fused with different effector domains (repressor or activator) for recruiting functional proteins to the specific genome loci, and then represses or activates the gene expression[31]. For instance, dCas9-VP64 (a transcription activator) and dCas9-p65AD (a single copy of the p65 activation domain) can efficiently activate reporter gene expression showing that CRISPR/Cas9 can act as a modular platform for transcription control[32]. Similarly, dCas9 can also be combined with epigenetic factors, such as histone-modifying/DNA methylation enzymes, for the epigenetic modification of genes. Cas9 can also be fused with fluorescent protein for DNA labeling of a specific region and can be used in chromosome imaging. Finally CRISPR/Cas9 orthologues were identified which increased the target specificity which is shown in Table 2. Distinct crRNAtracrRNA duplex and PAM requirements is imposed by many of the naturally occurring cas9 orthologues[30]. Hence, exploring orthologues Cas9 proteins with similar gRNA and PAM sequences would greatly expand the possible target sequences in a given genome and thus add new Cas 9 orthologues with unique properties in CRISPR/Cas system[33]. Cas9 proteins isolated from different bacterial species had unique and expanded PAM sequences that can aid in increasing on-target specificity. The alternative orthologous Cas9 requires different PAM sequences which increases the total number of target sites within a plant genome. CRISPR-Cpf1 is a class II, type V endonuclease developed from Prevotella and Francisella. Cpf1 uses single guide RNA (crRNA) 
complex for the cleavage, producing cohesive ends with 4-5 nucleotides 5' overhangs, with less or no off target events [34]. Similarly, C2C2 nuclease isolated from Leptotrichia shahii is capable of dual nuclease activity and can target single-stranded RNA[35]. In this way, CRISPR/Cas9 can be modified using multiple Cas9 orthologue-based platforms with different effectors like repressor, nuclease and transcription activator into the same cell, where they are guided by specific group of guide RNAs to carry out complex gene editing [33].

Table 1: CRISPR/Cas9 Modifications and their applications

\begin{tabular}{llc} 
Modification & Engineering & Application \\
\hline SpCas9n (Cas9n) & Substitution of aspartite to alanine (D10A) in the RuvC domain & Allows knock in via HDR \\
\hline Dead cas9 (dcas9) & Cas9 nuclease inactivation and double nicking using nickase & Nicking enhances specificity \\
\hline FokI Cas9 (fCas9) & Inactivated Cas9 nuclease fused with FokI nuclease & Increased on target activity \\
\hline
\end{tabular}

Table 2: CRISPR/Cas9 orthologues

\begin{tabular}{|c|c|c|c|c|c|}
\hline System & gRNA & Source & Protein & PAM $\left(5^{\prime}-3^{\prime}\right)$ & Reference \\
\hline CRISPR-cas 9 & tracrRNA+ crRNA & Streptococcus pyogenes & Cas9 & NGG & [10] \\
\hline CRISPR-cpf1 & Single RNA & Prevotella and Fracisella & Cas1, Cas2, Cas4 & YTN & [34] \\
\hline Ng-Ago & Single RNA & Natronobacterium gregoryi & Argonaute & Not required & {$[30]$} \\
\hline
\end{tabular}

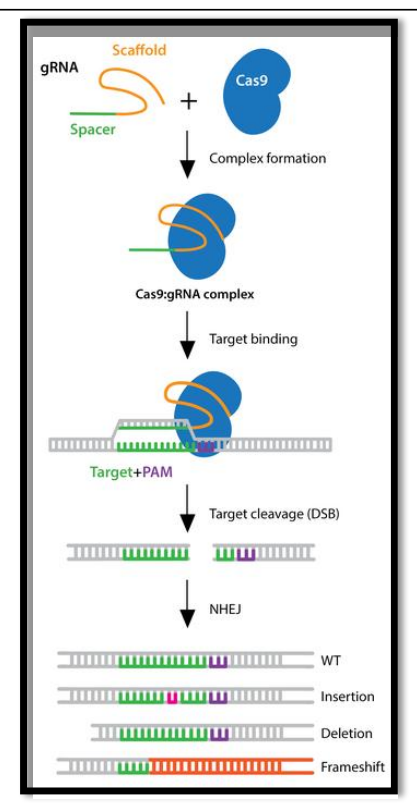

Fig: (a)
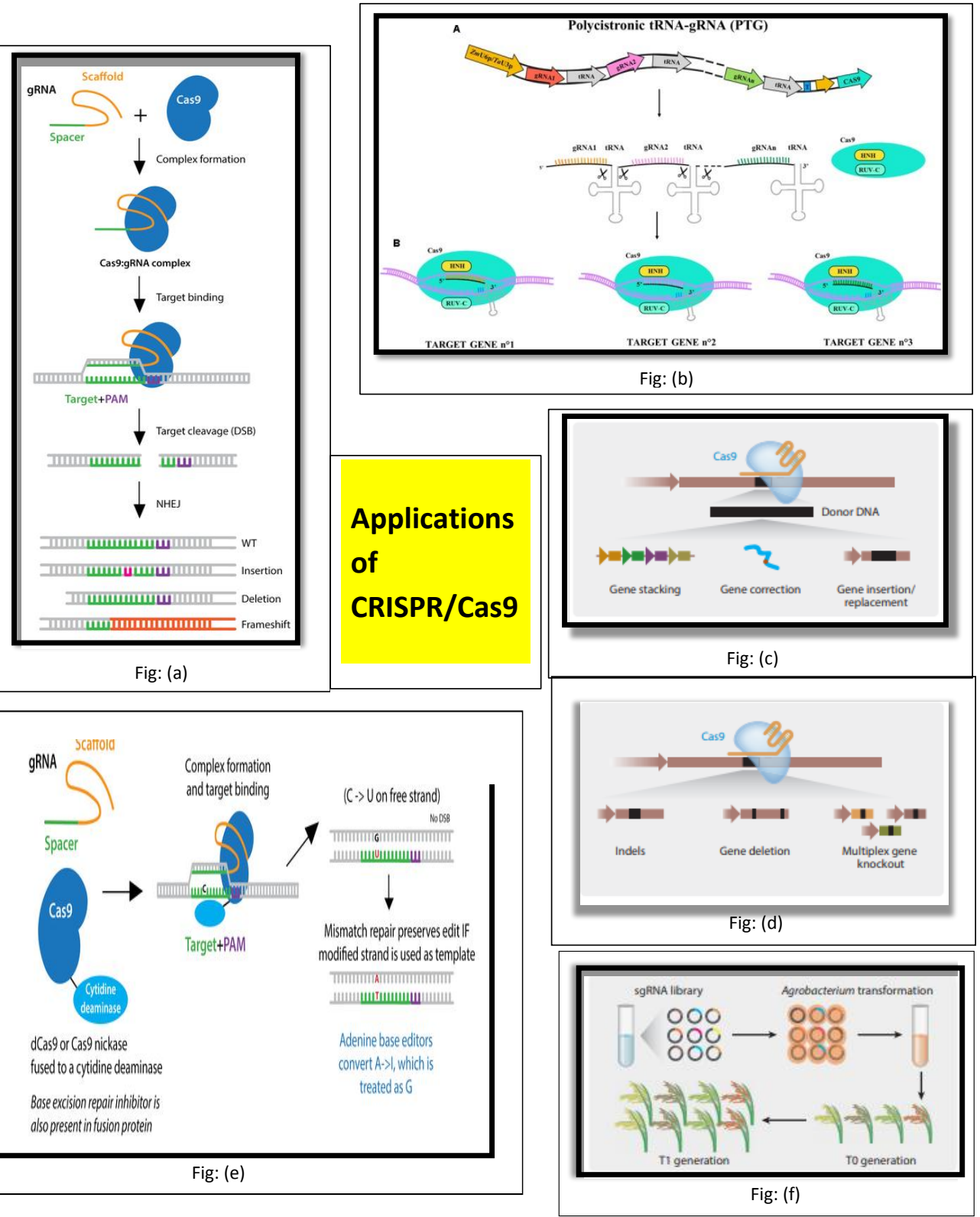

Figure 3: Applications of CRISPR/Cas9 [Fig(a):Genome editing using CRISPR/Cas9 through common pathway of DSB repair; Fig(b):Multiplexing using CRISPR/Cas9; Fig (c): Gene knock-in/replacement; Fig (d): Gene knockout using CRISPR/Cas9; Fig (e): Base editing; Fig (f) :High throughput mutant library] 


\subsection{Applications of CRISPR/Cas9 in crop improvement}

The simple, efficient and highly specific CRISPR/Cas9 system is a promising tool for genome editing and is expected to have larger impacts on plant biology and also on crop breeding. The elite cultivars can be precisely modified using genome editing technique, saving the time required compared to backcrossing in conventional breeding programs[39]. With the multiple traits being modified at a time, CRISPR/Cas9 system provides an efficient approach to pyramid breeding[40]. Gene editing by CRISPR/Cas9 has been adopted in nearly 20 crop species so far [41] for various traits including yield improvement, and biotic and abiotic stress management. NHEJ mediated gene editing is the most direct application of CRISPR/Cas9 gene editing. Biotic stress imposed by pathogenic micro-organisms account for more than $42 \%$ of potential yield loss and contributes to $15 \%$ of global declines in food production[42]. The negative regulators of disease resistance and grain development can be knocked out to obtain greater yield, host resistance against targeted pathogens and abiotic stresses like drought and salinity. In addition, CRISPR/Cas9 provides alternative approaches for delivering target genes into crops without any transgenic footprint, such as by viral infection, agro-infiltration, or preassembled Cas9 protein-sgRNA ribonucleoproteins transformation so as to bypass the traditional regulations on genetically modified organisms[43].

Table 3: Applications of CRISPR/Cas9 in agricultural crops

\begin{tabular}{|c|c|c|c|}
\hline Crop & Target gene & Target trait & References \\
\hline \multirow[t]{17}{*}{ Rice } & LAZY1 & Tiller-spreading & \multirow{17}{*}{$\begin{array}{l}{[43][50]} \\
{[51]} \\
{[52]} \\
{[45]} \\
{[53]} \\
{[54]} \\
{[49]} \\
{[55]} \\
{[56]} \\
{[57]}\end{array}$} \\
\hline & Gn1a, GS3, DEP1 & $\begin{array}{l}\text { Enhanced grain number, larger } \\
\text { grain size and dense erect } \\
\text { panicles }\end{array}$ & \\
\hline & SBEIIb & High amylose content & \\
\hline & OsERF922 & Enhanced rice blast resistance & \\
\hline & OsSEC3A & $\begin{array}{l}\text { Resistance to rice blast causing } \\
\text { pathogen Magnaporthe oryzae }\end{array}$ & \\
\hline & OSSWEET13 & Bacterial blight resistance & \\
\hline & OSMATL & Induction of haploid plants & \\
\hline & $A L S$ & Herbicide resistance & \\
\hline & EPSPS & Herbicide resistance & \\
\hline & 25604 gRNA for 12802 genes & $\begin{array}{l}\text { Creating genome wide mutant } \\
\text { library }\end{array}$ & \\
\hline & OsPDS, OsMPK2, OsBADH2 & $\begin{array}{l}\text { Involved in various abiotic } \\
\text { stress tolerance }\end{array}$ & \\
\hline & OSMPK2, OsDEP1 & Yield under stress & \\
\hline & $\begin{array}{l}\text { OsDERF1, OsPMS3, OsEPSPS, } \\
\text { OsMSH1, OsMYB5 }\end{array}$ & Drought tolerance & \\
\hline & OsMPK5 & $\begin{array}{l}\text { Various abiotic stress tolerance } \\
\text { and disease resistance }\end{array}$ & \\
\hline & $\begin{array}{l}\text { OsAOX1a, OsAOX1b,OsAOX1c, } \\
\text { OsBEL }\end{array}$ & Various abiotic stress tolerance & \\
\hline & OsHAK-1 & Low cesium accumulation & \\
\hline & OsPRX2 & Potassium deficiency tolerance & \\
\hline \multirow[t]{4}{*}{ Wheat } & $\begin{array}{lll}\text { TaMLOA1, } & \text { TaMLOB1 } & \text { and } \\
\text { TaMLOD1 } & & \\
\end{array}$ & Resistance to powdery mildew & \multirow{4}{*}{$\begin{array}{l}{[58]} \\
{[45],[59],[60]} \\
{[61]}\end{array}$} \\
\hline & GW2 & $\begin{array}{l}\text { Increased grain weight and } \\
\text { protein content }\end{array}$ & \\
\hline & EDR1 & Powdery mildew resistance & \\
\hline & TaGW2 & Incresase seed size & \\
\hline \multirow[t]{4}{*}{ Maize } & $W x 1$ & High amylopectin content & \multirow[t]{4}{*}{ [62], [63] } \\
\hline & TMSS & Thermosensitive male sterile & \\
\hline & $A L S$ & Herbicide resistance & \\
\hline & ARGOSB & Drought stress tolerance & \\
\hline \multirow[t]{5}{*}{ Tomato } & SIMLO1 & Powdery mildew resistance & \multirow[t]{5}{*}[8]{,$[46]$} \\
\hline & SIJAZ2 & Bacterial speck resistance & \\
\hline & $S P 5 G$ & Earlier harvest time & \\
\hline & SIAGL6 & Parthenocarpy & \\
\hline & SP, SP5G, CLV3, WUS, GGP1 & Tomato domestication & \\
\hline \multirow[t]{2}{*}{ Potato } & $A L S$ & Herbicide resistance & {$[29]$} \\
\hline & $W x 1$ & High amylopectin content & {$[64]$} \\
\hline Mushroom & $P P O$ & Anti-browning phenotype & [65] \\
\hline \multirow[t]{2}{*}{ Grapefruit } & CsLOB1 & Citrus canker resistance & [66] \\
\hline & CsLOB1 promoter & Alleviated citrus canker & [66] \\
\hline Orange & CsLOB1 promoter & Citrus canker resistance & [67] \\
\hline Cucumber & eIF4E & Virus resistance & {$[68]$} \\
\hline Camelina sativa & FAD2 & $\begin{array}{l}\text { Decreased } \\
\text { fatty acids }\end{array}$ & [69] \\
\hline Soybean & $A L S$ & Herbicide resistance & {$[70]$} \\
\hline Flax & $E P S P S$ & Herbicide resistance & {$[71]$} \\
\hline Cassava & EPSPS & Herbicide resistance & [72] \\
\hline
\end{tabular}

\subsection{Knockout mediated crop trait improvement}

Eliminating the negative traits that confer undesirable effects to the crop needs to be knocked out so as to obtain greater yield and biotic and abiotic stress tolerant crop variety. Hybrid breeding techniques and other important aspects of the crop breeding has been improved by knockout mechanism of gene editing[73].

\subsubsection{Increasing yield}

To cope up with the widespread food insecurity, the major sector to be think upon is crop yield. Yield is a complex trait governed by many factors. Knocking out negative regulators known to affect yield-determining factors such as grain number (OsGn1a), grain size (OsGS3), grain weight (TaGW2, OsGW5, OsGLW2 or TaGASR7), panicle size (OSDEP1, TaDEP1), tiller-spreading (LAZY1)and tiller number (OSAAP3) created the expected phenotypes in plants with loss-offunction mutations in these genes [44], [45], [74]. Simultaneous knockout of three grain weight-related genes (GW2, GW5, and TGW6) in rice led to trait pyramiding, which greatly increased grain weight[75]. M. Li et al., (2016) mutated the Gn1a, DEP1, and GS3 genes of the rice cultivar Zhounghua11 using CRISPR/Cas9, producing mutants with larger grain size, dense erect panicles and enhanced grain number. When Grain Weight 2 (GW2) gene in wheat is disrupted, it resulted in increased grain and protein content in wheat[77, p. 2]. However, because most yield-related traits are quantitative and controlled 
by quantitative trait loci, simply knocking out individual factors may not be sufficient to increase yield in the field. Huang et al., (2018) recently discovered a technique for large scale identification of genes that contributes to complex quantitative traits by combining pedigree analysis, whole-genome sequencing, and CRISPR/Cas9 technology[78]. The author sequenced 30 cultivars of the rice variety IR8 and selected 57 genes from high-yielding lines for gene editing via knockout or knockdown using Cas 9 or dCas9. This provided insight in crop yield development and may facilitate molecular breeding in rice[79].

\subsubsection{Increasing quality}

Crop quality can be improved by genome editing techniques. Till date, quality improvements by genome editing have been done for traits such as fragrance, longer keeping quality, improving oleic acid content, starch content etc. Fragrance is an important quality trait in rice, and fragrant rice varieties when cooked have increased commercial value. A defect in the betaine aldehyde dehydrogenase 2 (BADH2) gene results in the biosynthesis of 2-acetyl-1pyrroline, the major fragrance compound in fragrant rice. With the advent of convenient CRISPR/Cas9 techniques, scientists have recently added the fragrance trait to more than 30 elite rice cultivars in major planting areas of China [73]. CRISPR/Cas9 technology has been used to target the FAD2 gene in Camelina sativa, the emerging oil seed plant, to improve oleic acid content while decreasing polyunsaturated fatty acids [69]. Gluten proteins from cereal crops which trigger celiac disease are formed by $\alpha$-gliadin gene family. The CRISPR/Cas 9 genome editing tool offers a new way to alter traits controlled by large gene families with redundant functions. Indeed, by knocking out the most conserved domains of $\alpha$-gliadin family members, researchers have created low-gluten wheat[80]. CRISPR/Cas9 has been used in rice to generate the targeted mutations in SBEIIb, leading to higher proportion amylose, which improved the nutritional content and fine structure of starch [47]. Waxy maize with improved digestibility and higher bio-industrial application is prepared by DuPont Pioneer by knocking out the maize waxy gene (Wx1), which encodes Granule-bound starch synthase (GBSS) gene responsible for making amylose[81]. Development of parthenocarpic tomato fruits with huge market potential in processing industry was achieved simultaneously by two different groups. Klap et al., (2017) carried out knockout of Slagamous-like 6(SlAGL6) gene that otherwise severely hamper fertilization-dependent fruit set; made mutant plants capable of producing parthenocarpic fruits under heat stress conditions[82]. Alternatively, the other group has obtained parthenocarpic tomato fruits by mutating SIIAA9 gene involved in auxin signaling pathway that suppress the parthenocarpy[83]. By knocking out the gene responsible for polyphenol oxidase enzyme synthesis using CRISPR/Cas9 [65] developed a non-browning mushroom which triggers the post harvest quality and fetch higher prices.

\subsubsection{Increasing biotic and abiotic stress tolerance}

The yield loss caused by the disease causing pathogen and other abiotic stress is significantly higher. According to Ficke et al., (2017), the estimated yield loss due to plant pathogens is up to $16 \%$ [84]. So many attempts have been made in molecular biology to make disease resistant varieties. Y. Wang et al. (2014) has simultaneously edited three homoeoalleles TaMLOA1, TaMLOB1 and TaMLOD1 that confer heritable resistance to powdery mildew[85]. The CRISPR/Cas9 technology is used to generate Taedr1 wheat plants by simultaneous modification of the three homeologs of EDR1 which resulted in plants resistant to powdery mildew[86]. In rice, resistant varieties against blast disease and bacterial blight were obtained separately by mutagenesis of $O s E R F 922$, OsSEC3A and OsSWEET13 genes[56], [87], [88]. Further, powdery mildew and bacterial speck resistant tomato varieties were obtained by editing SIMLO1 and SIJAZ2 respectively[89], [90]. By the modification of CSLOB1 promoter, canker symptoms were alleviated in Duncan grapefruits[66]. The technology was further used to disrupt the coding region of $C s L O B 1$ resulting in no canker symptoms in Duncan grapefruit[67]. Chandrasekaran et al. (2016) conducted a research in cucumber by disrupting eIF4E (Eukaryotic translation initiation factor 4E), broad virus resistance was developed[68]. The plants were seen immune to cucumber Vein Yellowing virus (Ipomovirus) and were also resistant against potyviruses, Zucchini yellow mosaic virus and Papaya ring spot mosaic virus-W. CRISPR/Cas9 has also produced tungro disease resistant eif4g rice[91] and cotton leaf curl disease-resistant clcud cotton[92]. The destructive insect pests of rice i.e. plant hoppers and stem borers are the major yield reducing factors in rice. It was found that disrupting the 0 s $C Y P 71 A 1$ blocked serotonin biosynthesis and greatly increased salicylic acid levels, thereby confer resistance against these pests [93].

By developing slnpr1 mutant from isolated SINPR1 gene of tomato 'Ailsa Craig' using CRISPR/Cas9 resulted into drought tolerance in tomato[94]. Modification of OsPDS, OsMPK2, OsBADH2 genes in rice had led to increased tolerance to abiotic stresses[52]. Similarly Zhang et al. (2014) identified that modifications on OsDERF1, OsPMS3, OsEPSPS, OsMSH1, OSMYB5 genes lead to the drought tolerant varieties in rice[53]. Similarly, OsHAK-1 and OsPRX2 gene were edited in rice for obtaining low cesium accumulation and potassium deficiency tolerance respectively [55], [57]. CRISPR/Cas9 has been used to study the role of genes. Plant annexins which play a significant role in stress tolerance and plant development were tested using CRISPR/Cas9. Rice annexin gene (OsAnn3) was tested for its role in cold stress by OsAnn3 CRISPR knockouts [95].

\subsubsection{CRISPR/Cas9 for hybrid breeding}

An essential requirement for a high-quality hybrid variety is a male-sterile maternal parent. Using CRISPR/Cas-mediated gene knockout, tremendous progress has been made to produce male sterile lines. The thermosensitive genic male sterile 5 gene (TMS5) was knocked out in maize to generate male sterile line[96]. In similar ways thermosensitive male-sterile tms5 lines were developed in rice[97, p. 5], photosensitive genic male-sterile $c s a$ rice[98] and ms45 wheat were developed[99]. Recently, haploid rice was obtained by knockout of OsMATL by CRISPR/Cas9[100]. The selfish-gene suicide mechanism in rice caused by the toxic $O R F 2$ gene was knocked out improving the fertility of japonica-indica hybrids[101].

Similarly, CRISPR/Cas9 can be used by the breeders for domestication of wild varieties. Wild tomato accessions were introduced by targeting the coding sequences, cis-regulatory regions, and upstream open reading frames of genes associated with tomato morphology, flower and fruit production and ascorbic acid synthesis[102].

\subsection{Gene insertion and replacement}

CRISPR/Cas9 mediated gene insertion and replacement has mainly been used for developing herbicide resistant crop varieties. Substitution of key amino acids in the conserved domains of the endogenous acetolactate synthase $(A L S)$ and 5-enolpyruvylshikimate-3-phosphate synthase (EPSPS) can confer resistance to sulphonylurea-based herbicides[73]. Herbicide resistant rice variety was developed by utilizing several methods like disrupting DNA ligase 4 that is implicated by NHEJ repair[103], NHEJ mediated intron targeting[104], using two single-guide RNAs (sgRNAs) targeting the repair template [48] and the use of chimeric single-guide RNAs (cgRNAs) carrying both repair template sequences and target site[105]. Butler et al. (2016) produced herbicideresistant potatoes using geminivirus replicons that increase the copy number of CRISPR/Cas9 and a repair template[64]. Similarly, herbicide-resistant flax has been generated using a combination of single-stranded oligonucleotides and CRISPR/Cas9[71]. Recently, glyphosate tolerance variety of cassava was generated by a promoter swap and dual amino-acid substitutions achieved at the EPSPS locus[72]. In maize, grain yield under drought stress condition was increased by editing ARGOS8 gene[62]. When the GOS2 promoter was inserted into the 5'- untranslated region of the native ARGOS8 gene, or when it replaced the ARGOS8 promoter, increased ARGOS8 transcripts were detected that resulted in increased drought tolerance.

\subsection{Novel breakthroughs}

\subsection{Application in Base editing}

Many agronomically important traits are conferred by single-nucleotide polymorphism either in coding or non-coding sequences. This makes base editing quite useful in plant breeding and crop improvement. Genome wide-association studies have shown that single-base changes are responsible for the variations in elite traits in crops[106]. Hence, techniques for precise point mutations in crops are needed urgently. CRISPR/Cas9 mediated genome editing tool can be efficiently used for accurately converting one DNA base into another, without the use of a DNA repair template [107]. Base editing techniques requires the use of Cas9 nickase (ncas9) or dead cas9 (dcas9) fused to an enzyme with base conversion ablity. For example, cytidine deaminases convert cytosine to uracil which is treated as thymine in DNA repair or replication process, creating a C-G to T-A substitution[107], [108]. Likewise, adenine deaminases convert adenine to inosine, which is treated as guanine by polymerase, creating A-T to G-C substitutions[109]. Adeninne-deaminase-mediated base editing $(\mathrm{ABE})$ is more complicated that Cytidine-deaminase-mediated base editing as no known naturally occurring cytidine deaminases catalyze adenine deamination in DNA, rather than RNA, which was later made more efficient by the team of Gaudelli using several rounds of directed evolution and protein engineering[109]. 
One of the applications of base editing is to confer herbicide resistance. Sulfonylurea- or imidazolinone-resistant rice[46], Arabidopsis[110], and watermelon[111] have been generated by targeting $A L S$ with a plant cytidine base editor. CBE can also be used to produce nonsense mutations that disrupts the gene of interest and knockout their functions.CBE is highly specific than conventional SSN-mediated knockout, causing few if any indels[108]. HaloxyfopR-methyl resistant rice has been created by targeting acetyl-coenzyme-A carboxylase ACCase gene with a plant adenine base editor [112]. Using ABE fluorescence-tracking A to G base editor has been generated in rice[113]. Likewise, multiplex base editing was carried out using adenine base editor in rice[114]. In this way, base editing tool has given genome editing a new dimension, broadening its applications with nucleotide specific modifications at specific genomes.

\subsection{Transgene-free genome editing}

Conventional genome editing involves the delivery and integration of DNA cassettes encoding editing components into host genomes. The DNA cassettes then get degenerated but the fragments get incorporated which may produce undesirable effects[115]. The introduction of foreign DNA raises the regulatory issues regarding GM organisms. Side by side prolong expression of genome editing tools leads to the more off target activity due to the abundant nucleases in these organisms. This nature of conventional genome editing requires the introduction of DNA free genome editing tool which reduces the off target activity as well as escape the regulatory concerns from the national and international agencies[108]. DNA free genome editing was first conducted in Arabidopsis, tobacco, lettuce, and rice by transfection of CRISPR/Cas9 ribonucleoproteins (RNPs) into the plant protoplast[43]. Unfortunately, efficient and regenerable protoplasts system are not available for most of the agricultural crops which led to the particle bombardment mediated DNA free genome editing methods. CRISPR/Cas9 RNA and CRISPR/Cas9 RNPs have been delivered to wheat embryo by particle bombardment methods for obtaining genome editing[116], [117]. CRISPR/Cas9 RNPs has been used in maize not only to knockout mutants, but also to obtain targeted knock in mutants with the help of single-stranded DNA oligonucleotides[63].

\subsection{Multiplex Genome Editing using CRISPR}

The simultaneous targeting of several genes using a single molecular construct is multiplexing; which is the major advantage of CRISPR/Cas9 over its predecessors. Multiplexing has been reported in several crops by the assembly of multiple gRNAs under the control of a U3 or U6 promoter into a single construct[74],[118]. More recent gene editing methods involve exploitation of self cleavage capacity of RNA molecules containing tRNA sequences. The alternating sgRNA and tRNA sequences under the control of single U3 or U6 promoter permits reduction in size of the construct and thus limits the risk of silencing due to direct repetition of promoter sequences. The Polycistronic tRNA-gRNA (PTG) system uses such strategy to generate hereditable mutation in TaLpx-1 and TaMLO genes in hexaploid wheat[61]. The editing efficiency of PTG system was validated in wheat which makes it an effective tool for rapid trait pyramiding. Alternative to PTG systems, MGEs has been reported in rice, where crRNA transcription was obtained from introns inserted into Cpf1 and Cas9 sequences[119].

\subsection{High-throughput plant mutant libraries}

Whole genome scale library is an important tool for functional genomics. CRISPR/cas9 generated knockout mutant libraries has been constructed, where they targeted nearly 13,000 genes that are highly expressed in rice shoot base tissue and obtained more than 14,000 independent T0 lines[120]. Similarly, 34,234 genes in rice were targeted by another group and generated more than 90,000 transgenic plants[121]. Later on, immunity-associated leucine-rich repeat genes of subfamily XII, comprising 54 members in tomato were produced[122]

\subsection{Challenges regarding CRISPR/Cas9 tool}

Although genomes of many crop plants have been sequenced, the function of the vast majority of genes remains unknown. In other words, we have not reached the level of complete understanding of the functions of the major genes in agricultural crops. However, recent genome sequencing technology and genome-wide association studies (GWAS) allow us to predict the function of many genes, which confer resistance to biotic and abiotic stress, increase yield, quality etc. in cultivated crops. The field test after gene editing is necessary for correct evaluation of agronomic fitness. Most of the genes may have dual role in plant and may affect the physiology of plant along with altering the life cycle of pathogen, for example triple knockouts of wheat TaMLO were not only resistant to powdery mildew but also showed leaf chlorosis[85]

\subsection{Low efficiency of $\mathrm{HR}$}

Genome editing in the type-2 mode presents a considerable technical hurdle for regulators and ironically will probably be the most useful for plant breeding because it enables editing of existing alleles to redefine their function or new ones to be 'knocked-in'. NHEJ is the dominant DSBs repair mechanisms in many plant cell types and results in rapid DSBs repair or imprecise genome alteration. The delivery of repair templates is required in large amount by HDR to outcompete NHEJ which has always been a challenge. The delivery of DNA repair template has been improved using DNA replicons (deconstructed geminiviruses) but it has not been a perfect solution[31]. In that case an HR repair system, like Genome Repair Oligonucleotide technology, HDR boosters and NHEJ inhibitors are required[123], [124].

\subsection{Off-target effects}

When the CRISPR/Cas 9 complexes do not bind at the target, often a result of homologous sequences and/or mismatch tolerance, they will cleave off-target DSB and cause non-specific genetic modifications[36]. High frequency of off-target activity ( $\geq 50 \%)$ of RGEN (RNA-guided endonuclease)induced mutations at sites other than the intended on-target site is one major concern[125].

The most common type of cas9 protein is from the bacteria Streptococcus pyogenes (SpcAs9) which recognizes the NGC-type PAM[26]. This type of PAM sequence is widely distributed throughout plant genomes but it actually does not cover the entire plant genome so this may increase the off target cleavage due to complexing of gRNA with the mismatched complementary target DNA within the genome. Moreover, the CRISPR/Cas9 system accepts at least three mismatches in the $20 \mathrm{bp}$ DNA target sequence which increases the off target activity.

\subsection{Delivery of CRISPR/Cas9}

The greatest challenge to the implementation of CRISPR/Cas9 in agriculture is effective delivery of CRISPR/Cas9 machinery to right plant cells and subsequent regeneration or expression of viable plants. Traditional plant transformation systems including tissue culture and gene transfer remain preferred method for delivery but these are time-consuming, labour-intensive and produce random somatic mutations which renders inefficient[31] Moreover, many crops are recalcitrant to the regeneration through tissue culture. This creates an urgent need of novel delivery systems to achieve high efficiency genome editing in plants which may include use of regeneration boosters to enable tissue culture in recalcitrant species or even direct delivery to plant apical meristems or pollen grains to obtain edited plants without tissue culture[126].

Among all the challenges, public acceptance gets the greatest priority especially in case of agricultural commodities. As the foreign DNA may impose some side effects, modified crops have been regulated by the regulating authorities hindering the growth of CRISPR system.

\subsection{Future prospects}

Although much progress has been made in CRISPR/Cas9-based genome editing technology in the last few years, there are still some challenges left out: offtarget effects, side effects on nearby genes, mechanisms underlying the different effects of different sgRNAs on mutation efficiency, methods for efficient delivery in polyploid plants and regulatory concerns. Despite these challenges, the CRISPR based system will surely revolutionize and overcome most of the challenges. With the tremendous enthusiasm of the research community, genome editing technologies as represented by the CRISPR/Cas9 system will improve rapidly. This simple, affordable, and elegant genetic scalpel is expected to be widely applied to enhance the crop performance in the near future[32]

The CRISPR/Cas system has a great potential for improving the plant designs and exploit the potential of plant synthetic biology by inserting artificial DNA sequences, including promoters, genes, transcriptional regulatory elements and genome assemblies into the plant genomes. Nitrogen is a critical limiting 
factor for crop growth and development. CRISPR/Cas system could be used to transfer the genetic elements of the Nod factor signaling pathway from the legumes to cereals allowing the cereal crops to fix atmospheric nitrogen, which reduces our dependence on inorganic fertilizers.

Several attempts have been made for improving the environmental and disease tolerance of already domesticated crops. Instead, plant species that are already well adapted to different environments could be domesticated with high value traits. For example, the start-up Arvegenix is using the CRISPR/Cas tool to improve oil and meal quality in winter annual plant field pennycress. The goal is to make pennycress a cover crop that can be planted between two main growing seasons and a product similar to canola for oil and feed industry[31]. Next example of domestication by genome editing is tomato. A wild relative of tomato called ground cherry was edited and produced plants with higher yield and bigger fruit[127]. In the future, agro-biodiversity can be achieved and many problems associated with sustainable agriculture can be solved with the domestication of new crops with increased tolerance to a range of challenging environments, including deserts, maritime regions, low-nutrient soil, and cold climates.

Effective delivery of CRISPR/Cas9 machinery has always posed a major challenge to the CRISPR/Cas9 system. Current delivery systems are limited to specific plant species, genotypes and tissues. Thus, improved agrobacterium-mediated transformation may expand the range of delivery system[128] Beside this, almost all the present delivery systems use the laborious and time consuming tissue culture techniques. This requires the further innovation in achieving genotype-independent, tissue culture-free delivery via the plant germline or meristematic cells[129]. For example, use of pollen mediated transfer will overcome the limitations of species specificity and regeneration using pollination or artificial hybridization. Novel delivery systems using the nanotechnology like carbon nanotube mediated delivery also have great potential in expanding the applications of CRISPR/Cas9 as they may cause little cellular damage, have low toxicity and yield higher transformation efficiencies[130]

Off target activity of CRISPR/Cas9 is one of limitations hindering its applications. Thus several attempts and modifications have been done on Cas9 enzyme for achieving the target specificity and reduce the frequency of off target effects. Modification of cas 9 by the cas 9 nuclease inactivation and double nicking using nickase enhances the specificity [37]. Likewise, inactivated Cas9 nuclease fused with FokI nuclease also increases on target activity[38]. An increase in the protospacer adjacent motif length is a strategy that has been used to minimize the off-target cleavage. Improving the specificity of Cas9-linked base editors by extending sgRNA guide sequences, linking APOBEC1 with Cas9-HF1, and delivering base editors via RNPs could increase the applications of CRISPR/Cas9[131], [132].

Gene regulation, protein domain swaps and even new gene functions can be achieved in different scales with SDN-2 or SDN-3. Although, low frequency of HDR presents a considerable technical hurdle, increased efficiency can be obtained by the use of DNA replicons (deconstructed geminiviruses), but there is no clear indication of forthcoming breakthrough. Furthermore, Agrobacterium mediated delivery that uses type IV secretion system to deliver virulence effector proteins to plant cells and VirD2 protein covalently linked to the single-stranded T-DNA allows T-DNA transfer through protein transfer apparatus [133]; this mechanism could co-deliver CRISPR DNA or RNP with donor templates that stimulate HDR mediated genome editing. In mammalian cells simultaneous knockout of PolQ and genes essential for classical NHEJ (Ligase4, Ku70, Ku80) resulted in increased efficiency of HDR-mediated gene editing[134]. Same type of NHEJ inhibitors and HDR boosters can be used in plants too.

Target specific modifications using DNA free genome editing method could be effective so as to obtain non transgenic crops with transgenic method. The so obtained product could pass the regulatory concerns and gain public trust which increases the application of CRISPR in agriculture. Till now, what has been achieved with CRISPR technologies is just the tip of the iceberg. A sustainable future for agriculture can now be imagined using this new powerful genome editing tool. With that comes a responsibility to continue to resolve both the scientific and public concerns regarding its usage for a sustainable future[31]

\subsection{Conclusion and Recommendations}

Increase in world population from 7.3 billion to 9.7 billion by 2050 accompanied with increasing climate change has exacerbated the problem of food shortage. At the same moment, CRISPR/Cas9 has come at the right time when the conventional agriculture is striving to meet the growing food demands. This potential genome editing tool has provided scientists the ability to precisely and quickly insert the desired traits than other conventional breeding techniques. Application of genome editing tools in crop improvement to increase the yield, quality, biotic and abiotic stress tolerance and other traits will be a prominent area of work in the future. In the last 5 years, it is being applied vigorously in crops for functional genomics studies and combating biotic and abiotic stresses as well as to improve other important agronomic traits. Though several modifications to this technology have led to increase on-target efficiency, most work carried is preliminary and needs further improvement. Nevertheless, CRISPR/Cas9 based genome editing will gain popularity with time and be an essential technique to obtain 'suitably edited' plants that will help achieve the zero hunger goal i.e. second SDGs and maintain feed to the growing human population. Thus, advance molecular tool like CRISPR should be used in massive scale so as to make the world combat the global food challenges. Further researches should be done to overcome the challenges associated.

\section{References}

[1] J. L. Clarke and P. Zhang, "Plant biotechnology for food security and bioeconomy,” Plant Mol. Biol., vol. 83 (1), pp. 1-3, 2013.

[2] "FAOSTAT." [Online]. Available: http://www.fao.org/faostat/en/\#home. [Accessed: 07-May-2019].

[3] P. Stamm, R. Ramamoorthy, and P. P. Kumar, "Feeding the extra billions: strategies to improve crops and enhance future fo od security," Plant Biotechnol. Rep., vol. 5 (2), pp. 107-120, 2011.

[4] H.C.J. Godfray, “Food Security: The Challenge of Feeding 9 Billion People,” Science, vol. 327, (5967), pp. 812-818, 2010.

[5] J.D.G. Jones, “Elevating crop disease resistance with cloned genes,” Philos. Trans. R. Soc. B Biol. Sci., vol. 369 (1639), pp. $20130087-20130087,2014$.

[6] M.K. Samanta, A. Dey, and S. Gayen, “CRISPR/Cas9: an advanced tool for editing plant genomes,” Transgenic Res., vol. 25 (5), pp. 561-573, 2016.

[7] A. Ansari, “Engineered Dwarf Male-Sterile Rice: A Promising Genetic Tool for Facilitating Recurrent Selection in Rice,” Front. Plant Sci., vol. 8, 2017.

[8] D. Jaganathan, K. Ramasamy, G. Sellamuthu, S. Jayabalan, and G. Venkataraman, “CRISPR for crop improvement: An update review,” Front. Plant Sci., vol. 9, 2018.

[9] F. Georges and H. Ray, “Genome editing of crops: A renewed opportunity for food security,” GM Crops Food, vol. 8 (1), pp. 1-12, 2017.

[10] M. Jinek, K. Chylinski, I. Fonfara, M. Hauer, J. A. Doudna, and E. Charpentier, "A programmable dual-RNA-guided DNA endonuclease in adaptive bacterial immunity," Science, vol. 337 (6096), pp. 816-821, 2012.

[11] A. Malzahn, L. Lowder, and Y. Qi, “Plant genome editing with TALEN and CRISPR,” Cell Biosci., vol. 7, Pp. $21,2017$.

[12] E. Waltz, “With a free pass, CRISPR-edited plants reach market in record time,” Nat. Biotechnol., vol. 36 (1), pp. 6-7, 10 2018.

[13] G. Govindan and S. Ramalingam, "Programmable Site-Specific Nucleases for Targeted Genome Engineering in Higher Eukaryotes,” J. Cell. Physiol., vol. 231 (11), pp. 2380-2392, 2016.

[14] K. Chen and C. Gao, “TALENs: Customizable Molecular DNA Scissors for Genome Engineering of Plants,” J. Genet. Genomics, vol. 40 (6), pp. 271-279, 
2013.

[15] Y. Osakabe and K. Osakabe, “Genome Editing with Engineered Nucleases in Plants," Plant Cell Physiol., vol. 56 (3), pp. 389-400, 2015.

[16] K. Pauwels, N. Podevin, D. Breyer, D. Carroll, and P. Herman, “Engineering nucleases for gene targeting: safety and regulatory considerations," New Biotechnol., vol. 31 (1), pp. 18-27, 2014.

[17] Y. Ishino, M. Krupovic, and P. Forterre, "History of CRISPR-Cas from encounter with a mysterious repeated sequence to genome editing technology," J. Bacteriol., vol. 200 (7), pp. e00580-17, 2018.

[18] H. I. Ahmad, "A Review of CRISPR-Based Genome Editing: Survival, Evolution and Challenges.," Curr. Issues Mol. Biol., vol. 28, pp. 47-68, 2018.

[19] F. Jiang and J. A. Doudna, “CRISPR-Cas9 structures and mechanisms,” Annu. Rev. Biophys., vol. 46, pp. 505-529, 2017.

[20] P. Horvath and R. Barrangou, “CRISPR/Cas, the immune system of bacteria and archaea,” Science, vol. 327 (5962), pp. 167-170, 2010.

[21] X. Dai, X. Chen, Q. Fang, J. Li, and Z. Bai, “Inducible CRISPR genome-editing tool: classifications and future trends,” Crit. Rev. Biotechnol., vol. 38 (4), pp. 573-586, 2018.

[22] C.A. Lino, J.C. Harper, J.P. Carney, and J.A. Timlin, “Delivering CRISPR: a review of the challenges and approaches,” Drug Deliv., vol. 25 (1), pp. $1234-$ $1257,2018$.

[23] Y. Cui, J. Xu, M. Cheng, X. Liao, and S. Peng, "Review of CRISPR/Cas9 sgRNA design tools,” Interdiscip. Sci. Comput. Life Sci., vol. 10 (2), pp. 455-465, 2018.

[24] J. Li, “CRISPR/Cas: a novel way of RNA-guided genome editing: CRISPR/Cas: a novel way of RNA-guided genome editing,” Hered. Beijing, vol. 35 (11), pp. 1265-1273, 2013.

[25] H. Ledford, “CRISPR, the disruptor," Nat. News, vol. 522 (7554), p. 20, 2015.

[26] D. Zhang, Z. Li, and JF. Li, “Targeted gene manipulation in plants using CRISPR-Cas9.,” J. Genet. Genomics, 2016.

[27] C.M. Lee, T.J. Cradick, and G. Bao, “The Neisseria meningitidis CRISPR-Cas9 System Enables Specific Genome Editing in Mammalian Cells,” Mol. Ther., vol. 24 (3), pp. 645-654, 2016.

[28] F.A. Ran, P.D. Hsu, J. Wright, V. Agarwala, D.A. Scott, and F. Zhang, “Genome engineering using the CRISPR-Cas9 system,” Nat. Protoc., vol. 8 (11), Pp. $2281,2013$.

[29] S.R. Choudhury, Y. Cui, K. Lubecka, B. Stefanska, and J. Irudayaraj, "CRISPR-dCas9 mediated TET1 targeting for selective DNA demethylation at BRCA1 promoter," Oncotarget, vol. 7 (29), pp. 46545-46556, 2016.

[30] Y. Gao, X. Xiong, S. Wong, E.J. Charles, W.A. Lim, and L.S. Qi, “Complex transcriptional modulation with orthogonal and inducible dCas9 regulators,” Nat. Methods, vol. 13, Pp. 1043, 2016.

[31] C. Gao, “The future of CRISPR technologies in agriculture,” Nat Rev Mol Cell Biol, vol. 19 (5), pp. 275-276, 2018.

[32] G. Song, “CRISPR/Cas9: a powerful tool for crop genome editing," Crop J., vol. 4 (2), pp. 75-82, 2016.

[33] K. Yin, C. Gao, and J.L. Qiu, “Progress and prospects in plant genome editing," Nat. Plants, 2017.

[34] B. Zetsche, "Multiplex gene editing by CRISPR-Cpf1 using a single crRNA array," Nat. Biotechnol., vol. 35 (1), pp. 31-34, 2017.

[35] S.S.A. Zaidi, M.M. Mahfouz, and S. Mansoor, “CRISPR-Cpf1: A New Tool for Plant Genome Editing,” Trends Plant Sci., vol. 22 (7), pp. 550-553, 2017.

[36] L. Cong, “Multiplex Genome Engineering Using CRISPR/Cas Systems,” Science, vol. 339, (6121), pp. 819-823, 2013.

[37] P. Mali, “CAS9 transcriptional activators for target specificity screening and paired nickases for cooperative genome engineering," Nat. Biotechnol., vol. 31, Pp. 833, 2013.

[38] J.P. Guilinger, D.B. Thompson, and D.R. Liu, "Fusion of catalytically inactive Cas9 to FokI nuclease improves the specificity of genome modification," Nat. Biotechnol., vol. 32 (6), pp. 577-582, 2014.

[39] M. Wade, "High-throughput silencing using the CRISPR-Cas9 system: a review of the benefits and challenges," J. Biomol. Screen., vol. 20 (8), pp. 10271039, 2015.

[40] L. Bortesi and R. Fischer, "The CRISPR/Cas9 system for plant genome editing and beyond," Biotechnol. Adv., vol. 33 (1), pp. 41-52, 2015.

[41] A. Ricroch, P. Clairand, and W. Harwood, “Use of CRISPR systems in plant genome editing toward new opportunities in agriculture," Emerg. Top. Life Sci., vol. 1 (2), pp. 169-182, 2017.

[42] E.C. Oerke, “Crop losses to pests," J. Agric. Sci., vol. 144 (1), pp. 31-43, 2006.

[43] J.W.Woo, “DNA-free genome editing in plants with preassembled CRISPR-Cas9 ribonucleoproteins," Nat. Biotechnol., vol. 33, Pp. $1162,2015$.

[44] J. Miao, “Targeted mutagenesis in rice using CRISPR-Cas system," Cell Res., vol. 23 (10), Pp. 1233, 2013.

[45] Q. Shan, Y. Wang, J. Li, and C. Gao, “Genome editing in rice and wheat using the CRISPR/Cas system,” Nat. Protoc., vol. 9 (10), Pp. $2395,2014$.

[46] Z. Shimatani, "Targeted base editing in rice and tomato using a CRISPR-Cas9 cytidine deaminase fusion," Nat. Biotechnol., vol. 35 (5), Pp. $441,2017$.

[47] Y. Sun, “Generation of High-Amylose Rice through CRISPR/Cas9-Mediated Targeted Mutagenesis of Starch Branching Enzymes," Front. Plant Sci., vol. 8, 2017.

[48] Y. Sun, "Engineering herbicide-resistant rice plants through CRISPR/Cas9-mediated homologous recombination of acetolactate synthase," Mol. Plant, vol. 9 (4), pp. 628-631, 2016.

[49] R. Xu, “Gene targeting using the Agrobacterium tumefaciens-mediated CRISPR-Cas system in rice," Rice, vol. 7 (1), Pp. 5, 2014.

[50] H. Zhou, B. Liu, D.P. Weeks, M.H. Spalding, and B. Yang, "Large chromosomal deletions and heritable small genetic changes induced by CRISPR/Cas9 in rice," Nucleic Acids Res., vol. 42 (17), pp. 10903-10914, 2014.

[51] Y. Meng, “Targeted mutagenesis by CRISPR/Cas9 system in the model legume Medicago truncatula," Plant Cell Rep., vol. 36 (2), pp. $371-374,2017$. 
[52] Q. Shan, “Targeted genome modification of crop plants using a CRISPR-Cas system,” Nat. Biotechnol., vol. 31, Pp. 686, 2013.

[53] H. Zhang, "The CRISPR/C as9 system produces specific and homozygous targeted gene editing in rice in one generation," Plant Biotechnol. J., vol. 12 (6), pp. 797-807, 2014.

[54] K. Xie and Y. Yang, “RNA-Guided Genome Editing in Plants Using a CRISPR-Cas System,” Mol. Plant, vol. 6 (6), pp. 1975-1983, 2013.

[55] M. Nieves-Cordones, "Production of low-Cs + rice plants by inactivation of the K + transporter Os HAK 1 with the CRISPR -Cas system," Plant J., vol. 92 (1), pp. 43-56, 2017.

[56] J. Ma, "Disruption of OsSEC3A increases the content of salicylic acid and induces plant defense responses in rice," J. Exp. Bot., vol. 69 (5), pp. 1051$1064,2018$.

[57] X. Mao, "OsPRX2 contributes to stomatal closure and improves potassium deficiency tolerance in rice," Biochem. Biophys. Res. Commun., vol. 495 (1), pp. 461-467, 2018.

[58] T. Wang, J.J. Wei, D.M. Sabatini, and E.S. Lander, “Genetic screens in human cells using the CRISPR-Cas9 system," Science, vol. 343 (6166), pp. 80-84, 2014.

[59] J. Gil-Humanes, "High-efficiency gene targeting in hexaploid wheat using DNA replicons and CRISPR/Cas9," Plant J., vol. 89 (6), pp. $1251-1262,2017$.

[60] D. Kim, B. Alptekin, and H. Budak, “CRISPR/Cas9 genome editing in wheat,” Funct. Integr. Genomics, vol. 18 (1), pp. 31-41, 2018.

[61] W. Wang, “Transgenerational CRISPR-Cas9 Activity Facilitates Multiplex Gene Editing in Allopolyploid Wheat,” CRISPR J., vol. 1 (1), pp. 65-74, 2018.

[62] J. Shi, "ARGOS8 variants generated by CRISPR-Cas9 improve maize grain yield under field drought stress conditions," Plant Biotechnol. J., vol. 15 (2), pp. 207-216, 2017.

[63] S. Svitashev, C. Schwartz, B. Lenderts, J. K. Young, and A. Mark Cigan, "Genome editing in maize directed by CRISPR-Cas9 ribonucleoprotein complexes," Nat. Commun., vol. 7, Pp. 13274, 2016.

[64] N.M. Butler, N.J. Baltes, D.F. Voytas, and D.S. Douches, "Geminivirus-Mediated Genome Editing in Potato (Solanum tuberosum L.) Using SequenceSpecific Nucleases," Front. Plant Sci., vol. 7, 2016.

[65] E. Waltz, “Gene-edited CRISPR mushroom escapes US regulation,” Nat. News, vol. 532 (7599), Pp. 293, 2016.

[66] H. Jia, V. Orbovic, J.B. Jones, and N. Wang, "Modification of the PthA4 effector binding elements in Type I CsLOB1 promoter using Cas9/sgRNA to produce transgenic Duncan grapefruit alleviating Xcc $\Delta$ pthA4:dCsLOB1.3 infection," Plant Biotechnol. J., vol. 14 (5), pp. 1291-1301, 2016.

[67] H. Jia, "Genome editing of the disease susceptibility gene CsLOB1 in citrus confers resistance to citrus canker," Plant Biotechnol. J., vol. 15 (7), pp. 817823, 2017.

[68] J. Chandrasekaran, “Development of broad virus resistance in non-transgenic cucumber using CRISPR/Cas9 technology,” Mol. Plant Pathol., vol. 17 (7), pp. 1140-1153, 2016.

[69] W.Z. Jiang, I.M. Henry, P.G. Lynagh, L. Comai, E.B. Cahoon, and D.P. Weeks, "Significant enhancement of fatty acid composition in seeds of the allohexaploid, Camelina sativa, using CRISPR/Cas9 gene editing," Plant Biotechnol. J., vol. 15 (5), pp. 648-657, 2017.

[70] Y. Cai, “CRISPR/Cas9-mediated genome editing in soybean hairy roots," PLoS One, vol. 10 (8), p. e0136064, 2015.

[71] N. Sauer, J. Narvaez-Vasquez, J. Mozoruk, R. Miller, Z. Warburg, and M. Woodward, “Oligonucleotide-mediated genome editing provides precision and function to engineered nucleases and antibiotics in plants," Plant Physiol., 2017.

[72] A. Hummel, R. Chauhan, T. Cernak, A. Mutka, A. Vijayaraghavan, and A. Boyher, "Allele exchange at the EPSPS locus confers glyphosate tolerance in cassava," Plant Biotechnol. J., 2018.

[73] K. Chen, Y. Wang, R. Zhang, H. Zhang, and C. Gao, “CRISPR/Cas Genome Editing and Precision Plant Breeding in Agriculture,” Annu. Rev. Plant Biol., vol. 70 (1), pp. 667-697, 2019.

[74] W. Jiang, H. Zhou, H. Bi, M. Fromm, B. Yang, and D.P. Weeks, "Demonstration of CRISPR/Cas9/sgRNA-mediated targeted gene modification in Arabidopsis, tobacco, sorghum and rice," Nucleic Acids Res., vol. 41 (20), pp. e188-e188, 2013.

[75] R. Xu, "Rapid improvement of grain weight via highly efficient CRISPR/Cas9-mediated multiplex genome editing in rice," J. Genet. Genomics Yi Chuan Xue Bao, vol. 43 (8), pp. 529-532, 2016.

[76] M. Li, "Reassessment of the Four Yield-related Genes Gn1a, DEP1, GS3, and IPA1 in Rice Using a CRISPR/Cas9 System," Front. Plant Sci., vol. 7, 2016.

[77] Y. Zhang, "Analysis of the functions of TaGW2 homeologs in wheat grain weight and protein content traits," Plant J., vol. 94 (5), pp. 857-866, 2018.

[78] J. Huang, "Identifying a large number of high-yield genes in rice by pedigree analysis, whole-genome sequencing, and CRISPR-Cas9 gene knockout," Proc. Natl. Acad. Sci., vol. 115 (32), pp. E7559-E7567, 2018.

[79] J. Duitama, “Whole Genome Sequencing of Elite Rice Cultivars as a Comprehensive Information Resource for Marker Assisted Selection," PLoS ONE, vol. 10 (4), 2015.

[80] S. Sánchez-León, “Low-gluten, nontransgenic wheat engineered with CRISPR/Cas9,” Plant Biotechnol. J., vol. 16 (4), Pp. 902-910, 2018.

[81] "Pioneer Hi-Bred News Releases." [Online]. Available: https://www.pioneer.com/home/site/about/news-media/newsreleases/template.CONTENT/guid.1DB8FB71-1117-9A56-E0B6-3EA6F85AAE92. [Accessed: 09-May-2019].

[82] C. Klap, “Tomato facultative parthenocarpy results from SIAGAMOUS-LIKE 6 loss of function,” Plant Biotechnol. J., vol. 15 (5), pp. 634-647, 2017.

[83] R. Ueta, “Rapid breeding of parthenocarpic tomato plants using CRISPR/Cas9," Sci. Rep., vol. 7 (1), p. 507, 2017.

[84] A. Ficke, C. Cowger, G. Bergstrom, and G. Brodal, “Understanding Yield Loss and Pathogen Biology to Improve Disease Management: Septoria Nodorum Blotch - A Case Study in Wheat," Plant Dis., vol. 102 (4), Pp. 696-707, 2017.

[85] Y. Wang, "Simultaneous editing of three homoeoalleles in hexaploid bread wheat confers heritable resistance to powdery mildew," Nat. Biotechnol., vol. 32 (9), Pp. 947-951, 2014.

[86] Y. Zhang, "Simultaneous modification of three homoeologs of TaEDR1 by genome editing enhances powdery mildew resistance in wheat," Plant J., vol. 
91 (4), Pp. 714-724, 2017

[87] F. Wang, "Enhanced Rice Blast Resistance by CRISPR/Cas9-Targeted Mutagenesis of the ERF Transcription Factor Gene OsERF922," PLOS ONE, vol. 11 (4), p. e0154027, 2016.

[88] J. Zhou, "Gene targeting by the TAL effector PthXo2 reveals cryptic resistance gene for bacterial blight of rice," Plant J., vol. 82 (4), pp. 632-643, 2015.

[89] V. Nekrasov, C. Wang, J. Win, C. Lanz, D. Weigel, and S. Kamoun, "Rapid generation of a transgene-free powdery mildew resistant tomato by genome deletion," Sci. Rep., vol. 7 (1), Pp. 482, 2017.

[90] A. Ortigosa, S. Gimenez-Ibanez, N. Leonhardt, and R. Solano, "Design of a bacterial speck resistant tomato by CRISPR/Cas 9-mediated editing of SlJAZ2," Plant Biotechnol. J., vol. 17 (3), pp. 665-673, 2019.

[91] A. Macovei, "Novel alleles of rice eIF4G generated by CRISPR/Cas9-targeted mutagenesis confer resistance to Rice tungro spherical virus," Plant Biotechnol. J., vol. 16 (11), pp. 1918-1927, 2018.

[92] Z. Iqbal, M.N. Sattar, and M. Shafiq, “CRISPR/Cas9: A Tool to Circumscribe Cotton Leaf Curl Disease,” Front. Plant Sci., vol. 7, Pp. 475, 2016.

[93] H.P. Lu, "Resistance of rice to insect pests mediated by suppression of serotonin biosynthesis," Nat. Plants, vol. 4 (6), Pp. 338-344, 2018.

[94] R. Li, “CRISPR/Cas9-Mediated SINPR1 mutagenesis reduces tomato plant drought tolerance,” BMC Plant Biol., vol. 19 (1), p. $38,2019$.

[95] C. Shen, "Knock out of the annexin gene OsAnn3 via CRISPR/Cas9-mediated genome editing decreased cold tolerance in rice," J. Plant Biol., vol. 60 (6), pp. 539-547, 2017.

[96] J. Li, “Generation of thermosensitive male-sterile maize by targeted knockout of the ZmTMS5 gene," J. Genet. Genomics Yi Chuan Xue Bao, vol. 44 (9), Pp. 465-468, 2017.

[97] H. Zhou, "Development of Commercial Thermo-sensitive Genic Male Sterile Rice Accelerates Hybrid Rice Breeding Using the CRISPR/Cas9-mediated TMS5 Editing System," Sci. Rep., vol. 6, Pp. 37395, 2016

[98] Q. Li, “Development of japonica Photo-Sensitive Genic Male Sterile Rice Lines by Editing Carbon Starved Anther Using CRISPR/Cas9," J. Genet. Genomics Yi Chuan Xue Bao, vol. 43 (6), pp. 415-419, 2016.

[99] M. Singh, M. Kumar, M.C. Albertsen, J.K. Young, and A.M. Cigan, "Concurrent modifications in the three homeologs of Ms45 gene with CRISPR-Cas9 lead to rapid generation of male sterile bread wheat (Triticum aestivum L.)," Plant Mol. Biol., vol. 97 (4-5), pp. 371-383, 2018.

[100] L. Yao, “OsMATL mutation induces haploid seed formation in indica rice," Nat. Plants, vol. 4 (8), pp. 530-533, 2018.

[101] X. Yu, “A selfish genetic element confers non-Mendelian inheritance in rice," Science, vol. 360 (6393), pp. 1130-1132, 2018.

[102] T. Li, "Domestication of wild tomato is accelerated by genome editing," Nat. Biotechnol., Oct. 2018.

[103] M. Endo, M. Mikami, and S. Toki, “Biallelic Gene Targeting in Rice,” Plant Physiol., vol. 170 (2), pp. 667-677, 2016.

[104] J. Li, “Gene replacements and insertions in rice by intron targeting using CRISPR-Cas9,” Nat. Plants, vol. 2 (10), Pp. $16139,2016$.

[105] H. Butt, “Efficient CRISPR/Cas9-Mediated Genome Editing Using a Chimeric Single-Guide RNA Molecule," Front. Plant Sci., vol. 8, 2017.

[106] S. Henikoff and L. Comai, "Single-nucleotide mutations for plant functional genomics," Annu. Rev. Plant Biol., vol. 54, pp. 375-401, 2003.

[107] A.C. Komor, Y.B. Kim, M.S. Packer, J.A. Zuris, and D.R. Liu, "Programmable editing of a target base in genomic DNA without double-stranded DNA cleavage," Nature, vol. 533 (7603), pp. 420-424, 2016.

[108] Y. Zhang, K. Massel, I.D. Godwin, and C. Gao, “Applications and potential of genome editing in crop improvement," Genome Biol., vol. 19 (1), Pp. 210, 2018.

[109] N.M. Gaudelli, “Programmable base editing of A•T to G•C in genomic DNA without DNA cleavage," Nature, vol. 551 (7681), pp. 464-471, 2017.

[110] Y. Chen, Z. Wang, H. Ni, Y. Xu, Q. Chen, and L. Jiang, "CRISPR/Cas9-mediated base-editing system efficiently generates gain-of-function mutations in Arabidopsis," Sci. China Life Sci., vol. 60 (5), Pp. 520-523, 2017.

[111] S. Tian, "Engineering herbicide-resistant watermelon variety through CRISPR/Cas9-mediated base-editing," Plant Cell Rep., vol. 37 (9), Pp. $1353-$ 1356, 2018.

[112] C. Li, "Expanded base editing in rice and wheat using a Cas9-adenosine deaminase fusion," Genome Biol., vol. 19 (1), p. 59, 2018.

[113] F. Yan, "Highly Efficient A·T to G·C Base Editing by Cas9n-Guided tRNA Adenosine Deaminase in Rice," Mol. Plant, vol. 11 (4), Pp. 631-634, 2018.

[114] K. Hua, X. Tao, F. Yuan, D. Wang, and J.K. Zhu, “Precise A.T to G.C Base Editing in the Rice Genome,” Mol. Plant, vol. 11 (4), pp. 627-630, 2018.

[115] S. Kim, D. Kim, S.W. Cho, J. Kim, and J.S. Kim, "Highly efficient RNA-guided genome editing in human cells via delivery of purified Cas 9 ribonucleoproteins," Genome Res., vol. 24 (6), Pp. 1012-1019, 2014.

[116] Z. Liang, "Efficient DNA-free genome editing of bread wheat using CRISPR/Cas9 ribonucleoprotein complexes," Nat. Commun., vol. 8, Pp. $14261,2017$.

[117] Y. Zhang, "Efficient and transgene-free genome editing in wheat through transient expression of CRISPR/Cas9 DNA or RNA," Nat. Commun., vol. 7, Pp. 12617, 2016.

[118] H.L. Xing, “A CRISPR/Cas9 toolkit for multiplex genome editing in plants," BMC Plant Biol., vol. 14 (1), Pp. 327, 2014.

[119] D. Ding, K. Chen, Y. Chen, H. Li, and K. Xie, “Engineering Introns to Express RNA Guides for Cas9- and Cpf1-Mediated Multiplex Genome Editing," Mol. Plant, vol. 11 (4), Pp. 542-552, 2018.

[120] X. Meng, “Construction of a Genome-Wide Mutant Library in Rice Using CRISPR/Cas9,” Mol. Plant, vol. 10 (9), Pp. 1238-1241, 2017.

[121] Y. Lu, “Genome-wide Targeted Mutagenesis in Rice Using the CRISPR/Cas9 System,” Mol. Plant, vol. 10 (9), Pp. 1242-1245, 2017.

[122] T.B. Jacobs, N. Zhang, D. Patel, and G.B. Martin, “Generation of a Collection of Mutant Tomato Lines Using Pooled CRISPR Libraries," Plant Physiol., vol. 174 (4), pp. 2023-2037, 2017.

[123] N.A. Abdallah, C.S. Prakash, and A.G. McHughen, “Genome editing for crop improvement: Challenges and opportunities,” GM Crops Food, vol. 6 (4), Pp. 
183-205, 2016.

[124] H.D. Jones, “Challenging regulations: Managing risks in crop biotechnology,” Food Energy Secur., vol. 4 (2), Pp. 87-91, 2015.

[125] X.H. Zhang, L.Y. Tee, X.G. Wang, Q.S. Huang, and S.H. Yang, “Off-target Effects in CRISPR/Cas9-mediated Genome Engineering,” Mol. Ther. - Nucleic Acids, vol. 4, Pp. e264, 2015.

[126] J.H. Jung and Y.W. Seo, "Challenges in wide implementation of genome editing for crop improvement," J. Crop Sci. Biotechnol., vol. 20 (2), pp. 129135, 2017.

[127] Z.H. Lemmon, "Rapid improvement of domestication traits in an orphan crop by genome editing," Nat. Plants, vol. 4 (10), pp. 766-770, 2018.

[128] S.B. Gelvin, "Plant proteins involved in Agrobacterium-mediated genetic transformation," Annu. Rev. Phytopathol., vol. 48, pp. 45-68, 2010.

[129] Y. Ran, Z. Liang, and C. Gao, “Current and future editing reagent delivery systems for plant genome editing,” Sci. China Life Sci., vol. 60 (5), Pp. 490505, 2017.

[130] G.S. Demirer, "High aspect ratio nanomaterials enable delivery of functional genetic material without DNA integration in mature plants," Nat. Nanotechnol., vol. 14 (5), pp. 456-464, 2019.

[131] D. Kim, “Genome-wide target specificities of CRISPR RNA-guided programmable deaminases,” Nat. Biotechnol., vol. 35 (5), pp. 475-480, 2017.

[132] H.A. Rees, "Improving the DNA specificity and applicability of base editing through protein engineering and protein delivery," Nat. Commun., vol. 8, Pp. 15790, 2017.

[133] D.E. Voth, L.J. Broederdorf, and J.G. Graham, “Bacterial Type IV secretion systems: versatile virulence machines," Future Microbiol., vol. 7 (2), pp. 241 $257,2012$.

[134] P.A. Mateos-Gomez, F. Gong, N. Nair, K.M. Miller, E. Lazzerini-Denchi, and A. Sfeir, "Mammalian polymerase $\theta$ promotes alternative NHEJ and suppresses recombination," Nature, vol. 518 (7538), pp. 254-257, 2015. 